\title{
Mitochondrial Hyperacetylation in the Failing Hearts of Obese Patients Mediated Partly by a Reduction in SIRT3: The Involvement of the Mitochondrial Permeability Transition Pore
}

\author{
Elena C. Castillo José A. Morales ${ }^{\mathrm{a}}$ Héctor Chapoy-Villanueva ${ }^{\mathrm{a}}$ \\ Christian Silva-Platas ${ }^{a} \quad$ Niria Treviño-Saldaña ${ }^{a} \quad$ C. Enrique Guerrero-Beltrán ${ }^{a, b}$ \\ Judith Bernal-Ramírez ${ }^{a}$ Alejandro Torres-Quintanilla ${ }^{a}$ Noemí García \\ Keith Youker ${ }^{c}$ Guillermo Torre-Amionea,b,c Gerardo García-Rivas ${ }^{a, b}$
}

aTecnologico de Monterrey, Escuela de Medicina y Ciencias de la Salud, Medicina Cardiovascular y Metabolómica, Monterrey, México, 'ecnologico de Monterrey, Hospital Zambrano Hellion, TecSalud, Centro de Investigación Biomédica, San Pedro Garza García, México, 'Weill Cornell Medical College, Methodist DeBakey Heart \& Vascular Center, The Methodist Hospital, Houston, TX, USA

\section{Key Words}

Mitochondria • Acetylation • Sirtuins • Obesity • Biopsies • Heart failure

\begin{abstract}
Background/Aims: Cyclophilin D (CypD) mediates the mitochondrial permeability transition pore (mPTP) opening that contributes to mitochondrial dysfunction. CypD is regulated by its acetylation/deacetylation state that depends on Sirtuin-3 (SIRT3) mitochondrial deacetylase. Since obesity and metabolic syndrome decrease SIRT3 activity and expression, we tested the hypothesis that CypD hyperacetylation promotes mitochondrial dysfunction under this pathophysiological state, which is associated with ventricular dysfunction and heart failure. Methods: Myocardial tissue samples from patients with left ventricular heart failure, with either obesity or normal weight, were processed for the expression of SIRT3 and acetylation profile by Western Blot (WB). In addition, a rat model of obesity and metabolic syndrome induced by $30 \%(\mathrm{w} / \mathrm{v})$ of sucrose was conducted. The WB analysis was used to determine the levels of mitochondrial expression of SIRT3, Adenine Nucleotide Translocator (ANT), CypD and the acetylation profile, as well as immunoprecipitation to establish the acetylation levels of CypD. Mitochondrial function was assessed by oxygen consumption analysis and maximum $\mathrm{Ca}^{2+}$ retention capacity. Oxidative stress was assessed by aconitase activity, protein carbonyl
\end{abstract}

E. C. Castillo, J. A. Morales and H. Chapoy-Villanueva contributed equally to this work.

\begin{tabular}{ll}
\hline Gerardo García-Rivas & Tecnologico de Monterrey, Hospital Zambrano Hellion, \\
& San Pedro Garza García, PC 66278, Nuevo León (Mexico)
\end{tabular}

Tel. +52 (81) 8888 0472, Fax +52 (81) 8888 2223, E-Mail gdejesus@tec.mx 


\section{Cellular Physiology Cell Physiol Biochem 2019;53:465-479 \\ \begin{tabular}{ll|l} 
and Biochemistry & $\begin{array}{l}\text { DOl: 10.33594/000000151 } \\
\text { Published online: } 30 \text { August } 2019\end{array}$ & $\begin{array}{l}\text { C } 2019 \text { The Author(s). Published by } \\
\text { Cell Physiol Biochem Press GmbH\&Co. KG }\end{array}$
\end{tabular} \\ Castillo et al.: SIRT3 \& Acetylation in Failing Heart with Obesity}

and thiol groups content. Results: SIRT3 expression in the biopsies of the failing human hearts showed a $46 \%$ decrease in the expression levels of obese patients in comparison to the non-obese patients $(p=0.0219)$. Remarkably, body mass index was associated with protein acetylation (0.627; $p=0.035)$, suggesting that the acetylation profiles of the failing hearts of obese patients are partly mediated by a reduction in SIRT3, which is also associated with higher BNP levels, indicating a more severe ventricular dysfunction $(-0.636 ; p=0.043)$. Accordingly, obese rats demonstrated a SIRT3 mitochondrial expression decrease of $22 \%$ concomitantly with a hyperacetylated mitochondrial profile, including CypD. Cardiac mitochondria from obese animals were 2.5 -fold more prone to MPTP opening than the controls. Conclusion: Our results indicate that obesity reduces SIRT3 expression and that CypD hyperacetylation increases mPTP opening, suggesting that the activation of SIRT3 might be a potential target to decrease ventricular dysfunction and slow the progression of heart failure.

(C) 2019 The Author(s). Published by

Cell Physiol Biochem Press GmbH\&Co. KG

\section{Introduction}

Heart failure (HF) occurs more frequently among people with obesity, diabetes mellitus, and metabolic syndrome (MetS) [1]. Obesity and MetS are associated with altered cardiac mitochondrial activity, which is believed to contribute to the impairment of ventricular functions [2]. Many studies of rodents and humans with HF have revealed a reduced ability to enhance fatty acid oxidation in the face of increased lipid delivery as well as metabolic inflexibility, which may be consistent with uncoupled myocardial oxygen consumption $\left(\mathrm{MVO}_{2}\right)$, energy depletion, and decreased contractile function [3]. The mechanisms for reduced cardiac efficiency are partially understood, but it has been suggested that mitochondrial dysfunction and subsequent cell death, particularly due to the opening of the mPTP, are involved [4,5]. Triggering factors for mPTP, such as oxidative stress and $\mathrm{Ca}^{2+}$ overload, are present in cardiomyocytes affected by obesity and MetS. Although the precise components of MPTP are still being elucidated, the role of cyclophilin D (CypD) as an inductor of the MPTP opening has been well-documented [6]. The physiological function of CypD is not well-known, however CypD binds to the mitochondrial inner membrane components, such as adenine nucleotide translocator (ANT) [7] and ATP Synthase [8], which induces the opening of mPTP. The binding of CypD to the inner membrane components is positively correlated to its acetylation state in the mitochondrial matrix. In this context, it has been demonstrated that SIRT3, a class III NAD ${ }^{+}$-dependent deacetylase, diminishes the activity of CypD, inducing its dissociation from ANT and thus avoiding the mPTP opening [9]. Furthermore, cardiomyocytes from a SIRT3 knockout murine model showed an agedependent mitochondrial swelling associated with cardiac hypertrophy, fibrosis, and hypersensitivity to cardiac stress. In turn, mitochondrial hyperacetylation is associated with a SIRT3 deficiency that accelerates the development of MetS [10, 11], increasing hyperlipidemia and obesity. Furthermore, it has been demonstrated that SIRT3 plays an important role in vascular homeostasis, having a direct effect on insulin-induced mesenteric vasorelaxation that leads to vascular dysfunction in obese animals [12]. Murine models have shown that chronic changes in metabolism induce SIRT3 downregulation and is associated with impaired mitochondrial activity and MPTP opening [10-12]. In addition, acute damage by myocardial infarction induced SIRT3 downregulation in diabetic rats [13]. However, it has not been demonstrated that SIRT3 downregulation alone by chronic metabolic changes has a pathophysiological role by inducing cardiac cell dysfunction and energetic impairment leading to decreased cardiac function and the development of heart failure. Moreover, the relevance in human heart failure has not been studied.

In the present study we show that development of MetS is accompanied by cardiac mitochondrial sensitivity to the MPTP underlying a SIRT3- dependent CypD hyperacetylation mechanism, which contributes to energetic failure and cardiac dysfunction in obese rats. These findings correlate with the changes to the acetylation profile in the end-stage failing hearts of patients with obesity. Overall, this work highlights the importance of SIRT3 


\section{Cellular Physiology Cell Physiol Biochem 2019;53:465-479

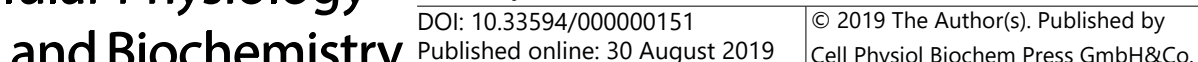 \\ Castillo et al.: SIRT3 \& Acetylation in Failing Heart with Obesity}

regulation in preventing heart diseases related to mitochondrial dysfunction in patients with obesity and MetS.

\section{Materials and Methods}

Population of patients with left ventricular heart failure ( $\mathrm{LV}-\mathrm{HF}$ )

Myocardial tissue samples were collected at the time of cardiac transplantation or LVAD implantation that did not have a primary diagnosis of myocarditis as defined by the Dallas criteria, postpartum cardiomyopathy (CM), congenital CM, hypertrophic CM, and Adriamycin induced CM from 2000 to 2010 at the Methodist Hospital. Tissue samples were taken from the left ventricular (LV) apex or LV free wall without adipose tissue and avoiding scar tissue. The investigation conforms with the principles outlined in the Declaration of Helsinki (Br Med J 1964; ii: 177). All samples were collected under an IRB approved protocol (IRB(3N)0511-0100). A waiver of consent was obtained due to the collection of normally discarded tissues which are coded at the time of collection with no identifying information.

Immunodetection of SIRT3, ANT, CypD, and acetylation profile

Expression levels of SIRT3 (Cell signaling technology. Massachusetts, USA), ANT (Abcam. Massachusetts, USA) and CypD (Abcam), as well as the acetylation protein profile (Cell signaling technology), were conducted using the conditions recommended by the manufacturers. To detect HRP-labeled secondary antibodies, the Clarity Western ECL Substrate was employed (Bio-Rad. Philadelphia, USA) in a UVP Chimio System (BioSpectrum 415 Imaging System. Cambridge, UK). GAPDH (Abcam) expression was used as control for total human heart tissue samples and VDAC expression (Abcam) for mitochondrial heart tissue from rats.

\section{Immunoprecipitation}

Cardiac mitochondria ( $0.5 \mathrm{mg}$ ) were solubilized in immunoprecipitation buffer $(150 \mathrm{mM} \mathrm{NaCl}$, EGTA 1 $\mathrm{mM}$, Igepal 1\%, Tris-HCl $20 \mathrm{mM} \mathrm{pH}$ 7.2, protease inhibitor cocktail), clarified of endogenous IgG, and incubated with 2 ug of anti-CypD or the isotype IgG as control for 1 hour at $4^{\circ} \mathrm{C}$ in a rotator. The immunoprecipitation complexes were captured by adding Protein G sepharose beads (50\% activated slurry) to the solubilized protein and incubated as mentioned above, overnight. Beads were sedimented by centrifugation, washed $\mathrm{X} 3$ and the complexes were eluted in SDS-loading buffer prior to electrophoretic separation and subsequent western blot analysis.

\section{Obesity and MetS induction and characterization}

Experimental procedures were approved by the animal use and care committee of the Medical School of Tecnologico de Monterrey (protocol:2011-Re-013). All reagents were obtained from Sigma-Aldrich (Sigma-Aldrich. Missouri, USA) unless otherwise stated.

Male Wistar rats 8 to 10 weeks old were used (animal used:112). Obesity and MetS were induced by the inclusion of $30 \%(\mathrm{w} / \mathrm{v})$ sucrose in their drinking water for either 6 or 12 months. Age-matched rats given normal drinking water were used as controls (CTL). Mean arterial pressure was measured by the indirect tailcuff method (CODA Monitor System; Kent Scientific. Connecticut, USA) while cardiac function was evaluated by echocardiography as previously described [14]. For these measures, animals were anesthetized with pentobarbital sodium ( $40 \mathrm{mg} / \mathrm{kg}$, ip). After overnight fasting, serum triglycerides, glucose and insulin were determined. Homeostatic model assessment (HOMA) of insulin resistance (IR) was obtained by the formula HOMA-IR=(insulin $x$ glucose)/22.5. At the end of each treatment, animals were sacrificed by an injection of a lethal dose $(80 \mathrm{mg} / \mathrm{kg}$, ip) of sodium pentobarbital. Abdominal adipose tissue values were normalized with the right femur weight. Additionally, cardiac mechanical function was evaluated in isolated hearts as described previously [15]. Baseline was established during 5 minutes of Krebs-Henseleit perfusion and was followed by perfusion of isoprenaline (ISO). $\mathrm{MVO}_{2}$ and the mechanical performance index (MPI) were obtained from the last $3 \mathrm{~min}$ of contraction at each ISO concentration. MPI was defined as the product of $\mathrm{LV}$ developed pressure $\times \mathrm{HR}(\mathrm{mmHg} X$ heart beats $\mathrm{X}$ min- 1$)$. At the end of the stimulation the hearts were released by cutting through the aorta and frozen with liquid nitrogen, weighted and stored at $-80^{\circ} \mathrm{C}$. ATP and phosphocreatine levels were measured using a dual-pump gradient HPLC [15]. 


\section{Cellular Physiology Cell Physiol Biochem 2019;53:465-479 \\ \begin{tabular}{l|l|l} 
DOI: 10.33594/000000151 & ( 2019 The Author(s). Published by
\end{tabular} \\ and Biochemistry Published online: 30 August 2019 Cell Physiol Biochem Press GmbH\&Co. KG \\ Castillo et al.: SIRT3 \& Acetylation in Failing Heart with Obesity}

RNA isolation, $c D N A$ synthesis, and real-time reverse transcription polymerase chain reaction (RT-qPCR) amplification

Apical heart fraction was isolated using the TRIzol Reagent (Invitrogen, USA) to perform a real-time reverse transcriptase PCR analysis (RT-qPCR) of GCN5L1 (general control of amino acid synthesis 5-like 1) in the Rotor-Gene 3000 (Corbett Research. Concorde, Australia). Samples were subjected to $0.5 \mu \mathrm{L}$ of DNase treatment (Agilent Technologies, Inc. California, USA). cDNA was synthesized from $1 \mu \mathrm{g}$ RNA using the AffinityScript cDNA Synthesis Kit (Agilent Technologies). Hypoxanthine phosphoribosyltransferase-1 (HPRT-1) mRNA was used as an endogenous control (Supplementary Table S1 - for all supplemental material see www.cellphysiolbiochem.com). The qPCR was performed with the Brilliant III Ultra-Fast SYBR Green QPCR Master Mix (Agilent Technologies). The analysis was carried out by the $\Delta \Delta \mathrm{C}_{\mathrm{T}}$ method. Results were expressed as the difference of the relative expression level data $\left(2^{-\Delta \Delta T}\right)$.

\section{Mitochondria isolation and assays}

Ventricular tissue was minced and homogenized in Sucrose-HEPES-EDTA isolation medium (Sucrose $0.25 \mathrm{M}$, HEPES $10 \mathrm{mM}$ and EDTA $1 \mathrm{mM}, \mathrm{pH}$ 7.4). The mitochondrial fraction was obtained by differential centrifugation using the Subtilisin A protease and resuspended in Sucrose-HEPES medium as previously described [16]. Lowry method was used for protein quantification.

\section{Mitochondrial oxygen consumption}

For oxygen consumption analysis, $0.5 \mathrm{mg}$ of mitochondria were incubated in $1 \mathrm{~mL}$ of standard reaction medium (in mM: $125 \mathrm{NaCl}, 10$ HEPES and $3 \mathrm{KH}_{2} \mathrm{PO}_{4}, \mathrm{pH}$ 7.2). Respiration states were evaluated using 10 mM glutamate-malate, $100 \mathrm{nM}$ ADP, and $40 \mathrm{nM}$ carbonyl cyanide m-chlorophenylhydrazone (CCCP). State 4 respiration was evaluated in the presence of glutamate-malate and CCCP-stimulated respiration while the state 3 respiration was measured after addition of ADP. Mitochondrial oxygen consumption was measured using the Clark-type oxygen electrode, YSI 5300A Biological Oxygen Monitor (YSI Life Sciences. Ohio, USA).

\section{Mitochondrial $\mathrm{Ca}^{2+}$ uptake and release}

Opening of mPTP was evaluated in isolated mitochondria using $\mathrm{Ca}^{2+}$, and the maximum $\mathrm{Ca}^{2+}$ retention capacity (CRC) and the half time of retention in standard reaction medium were measured with the metallochromic indicator, Arsenazo III for $45 \mathrm{~min}$ and ended with CCCP using $20 \mathrm{mM}$ glutamate-malate, as a substrate as previously described [17]. To evaluate maximum CRC, $10 \mathrm{nM} \mathrm{Ca}^{2+}$ was added incrementally

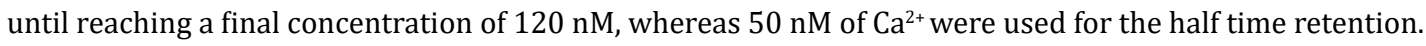
The assays were evaluated at $28^{\circ} \mathrm{C}$ using a Synergy HT detection microplate reader (BioTek Instruments, Vermont, USA). As a negative control, mitochondria were incubated with $\mathrm{Ca}^{2+}$ and $0.2 \mu \mathrm{M}$ cyclosporine A (CsA). The involvement of ANT and CypD in the opening of MPTP was evaluated using ADP, carboxyatractyloside (CAT) and CsA respectively.

\section{Oxidative stress markers}

Mitochondrial aconitase activity was determined spectrophotometrically, monitoring the NADP $^{+}$ reduction $(340 \mathrm{~nm})$ by isocitrate dehydrogenase. The reaction was carried out in $0.1 \mathrm{ml}$ of the medium that contained: $120 \mathrm{mM} \mathrm{KCl}, 5.0 \mathrm{mM} \mathrm{KH}_{2} \mathrm{PO}_{4}, 3.0 \mathrm{mM}$ HEPES, $1.0 \mathrm{mM}$ EGTA, $1.0 \mathrm{mM} \mathrm{MgCl}{ }_{2}, 0.01 \% \mathrm{Tx}-100, \mathrm{pH}$ 7.2 followed by addition of: $1.0 \mathrm{mM}$ sodium citrate, $0.2 \mathrm{mM} \mathrm{NADP}^{+}$, and $1 \mathrm{U} / \mathrm{mL}$ isocitrate dehydrogenase. Protein carbonyl content was determined by the formation of dinitrophenyl (DNP) hydrazone adducts from the derivatization of protein carbonyl groups with 2, 4-dinitrophenylhydrazine (DNPH) detected at $375 \mathrm{~nm}$. Thiol groups on ANT were measured by eosin 5-maleimide (EMA), which preferentially attacks the Cys159 of the cysteine thiols of ANT susceptible to oxidation. Briefly, the mitochondrial protein was incubated following the free thiol content conditions assay. After incubation, EMA ( $20 \mathrm{nmol} / \mathrm{mg}$ protein) was added and incubated $30 \mathrm{~min}$ in presence or absence of $\mathrm{Ca}^{2+}$. The reaction was stopped by $2 \mathrm{mM}$ DTT and then centrifuged at 14,000 rpm for $30 \mathrm{~min}$. The pellet was mixed in Laemmli buffer (without $\beta$-mercaptoethanol) for SDS/PAGE, and ANT-EMA reaction was visualized by the UVP Chimio System.

\section{Statistical analysis}

The data are shown as the mean \pm the standard error. The statistical analysis was performed by the Graph Pad Instat program (Graph Pad Software Inc. California, USA). Student's t-test was used to compare 


\section{Cellular Physiology Cell Physiol Biochem 2019;53:465-479 \\ \begin{tabular}{ll|l} 
and Biochemistry $10.33594 / 000000151$ & $\begin{array}{l}\text { Dublished online: } 30 \text { August } 2019 \\
\text { Pu } 2019 \text { The Author(s). Published by } \\
\text { Cell Physiol Biochem Press GmbH\&Co. KG }\end{array}$ \\
\hline
\end{tabular} \\ Castillo et al.: SIRT3 \& Acetylation in Failing Heart with Obesity}

between the obese/MetS (OB) and CTL group. For association analyses of variables in human HF linear regression or Spearman correlation tests were used. Ac-lys values were log transformed for normalization and group comparison by body max index (BMI) was performed with Student's t -test.

\section{Statement of Ethics}

Subjects have given their written informed consent. The study protocol has been approved by the research institute's committee on human research. Animal experiments conform to internationally accepted standards and have been approved by the appropriate institutional review body.

\section{Results}

Hyperacetylation and reduced SIRT3 expression in obese HF patients

We investigated the acetylation profile and SIRT3 protein levels in human HF biopsies retrieved from each patient's left ventricle at the time of LVAD implantation or transplantation. The patients' clinical characteristics are shown in Table 1. We subdivided this cohort of patients with end-stage HF in accordance to the BMI of non-obese patients and patients with obesity. The non-obese patients were $80 \%$ male and $40 \%$ Caucasian. For the patients with obesity, the mean EF was similar $(21.83 \pm 3.4 \%)$, but the BMI was $34.39 \pm 1.49$ (p $=0.0001)$. In this cohort of patients, we found that the SIRT3 expression levels in the HF patients with a BMI $>30$ were $46 \%$ down regulated $(\mathrm{p}=0.0219)$ (Fig. $1 \mathrm{~A})$, whereas protein acetylation was increased (Fig. 1B). The associated coefficients between SIRT3 and acetylation profile were negatively correlated $(-0.664 ; \mathrm{p}=0.023$; Fig. $1 \mathrm{C})$, whereas BMI values correlated positively with protein acetylation (0.627; $\mathrm{p}=0.035$; Fig. 1D). Interestingly, lower SIRT3 levels were associated with higher BNP levels (-0.636; $p=0.043$; Fig. 1E), which are a relevant marker in left ventricular dysfunction. These data suggest that the acetylation profile of the failing hearts of patients with obesity is partly mediated by a reduction in SIRT3.

Obesity and MetS impair cardiac contractility in sucrose-fed rats

In order to determine the contribution of cardiac SIRT-dependent hyperacetylation to cardiac dysfunction in patients with obesity, we used a sucrose-fed rat model. Consistent with obesity and MetS model [17], we observed a significant increase in total body weight, and a 2-fold increaseinabdominal adipose tissuein both groupsincomparison totheircorresponding controls (Table 2). Blood analyses showed a 2-fold increase in plasma triglycerides and circulating levels of insulin. HOMA ratio increases ( 2 and 2.7-fold, respectively) in the 6-and 12 -months obese group, when compared to the control groups, indicating insulin resistance. However, glucose levels did not change significantly. An elevated mean arterial pressure at 6and 12-months in the obese groups was also observed. To assess whether these metabolic changes had cardiac consequences, we performed an echocardiography analysis and observed that diastolic function was modified (Table 3); the 6-month obese rats showed an increase in A-wave values of $30 \% \quad(\mathrm{p}<0.05)$, with a $21 \% \quad(\mathrm{p}<0.0001)$ reduction in the $\mathrm{E} / \mathrm{A}$ ratio and a deceleration time increase of $21 \%(p<0.05)$

Table 1. Demographic data. Mean \pm SEM. Mann-Whitney test. EF: Ejection fraction; LVEDD = left ventricular end-diastolic diameter; LVESD: left ventricular end-systolic diameter

\begin{tabular}{lccc}
\hline Demographics & Non-Obese (NOB) & Obese (OB) & p-value \\
\hline Number & 5 & 6 & \\
Age (year) & $55 \pm 15$ & $55 \pm 8$ & \\
BMI & $23.8 \pm 0.4$ & $34.4 \pm 1.5$ & $0.0001^{* * *}$ \\
Gender (\%) & & & \\
Male & 80 & 100 & \\
Female & 20 & 0 & \\
Race & & & \\
Caucasian & 2 & 4 & \\
African American & 2 & 2 & \\
Other & 1 & 0 & \\
Echocardiography & & & \\
EF (\%) & $27.8 \pm 9.2$ & $21.8 \pm 3.4$ & 0.5297 \\
LVEDD (mm) & $65.2 \pm 7.8$ & $67.0 \pm 2.6$ & 0.8317 \\
LVESD (mm) & $55.3 \pm 15.3$ & $57.3 \pm 5.4$ & 0.8985 \\
\hline
\end{tabular}




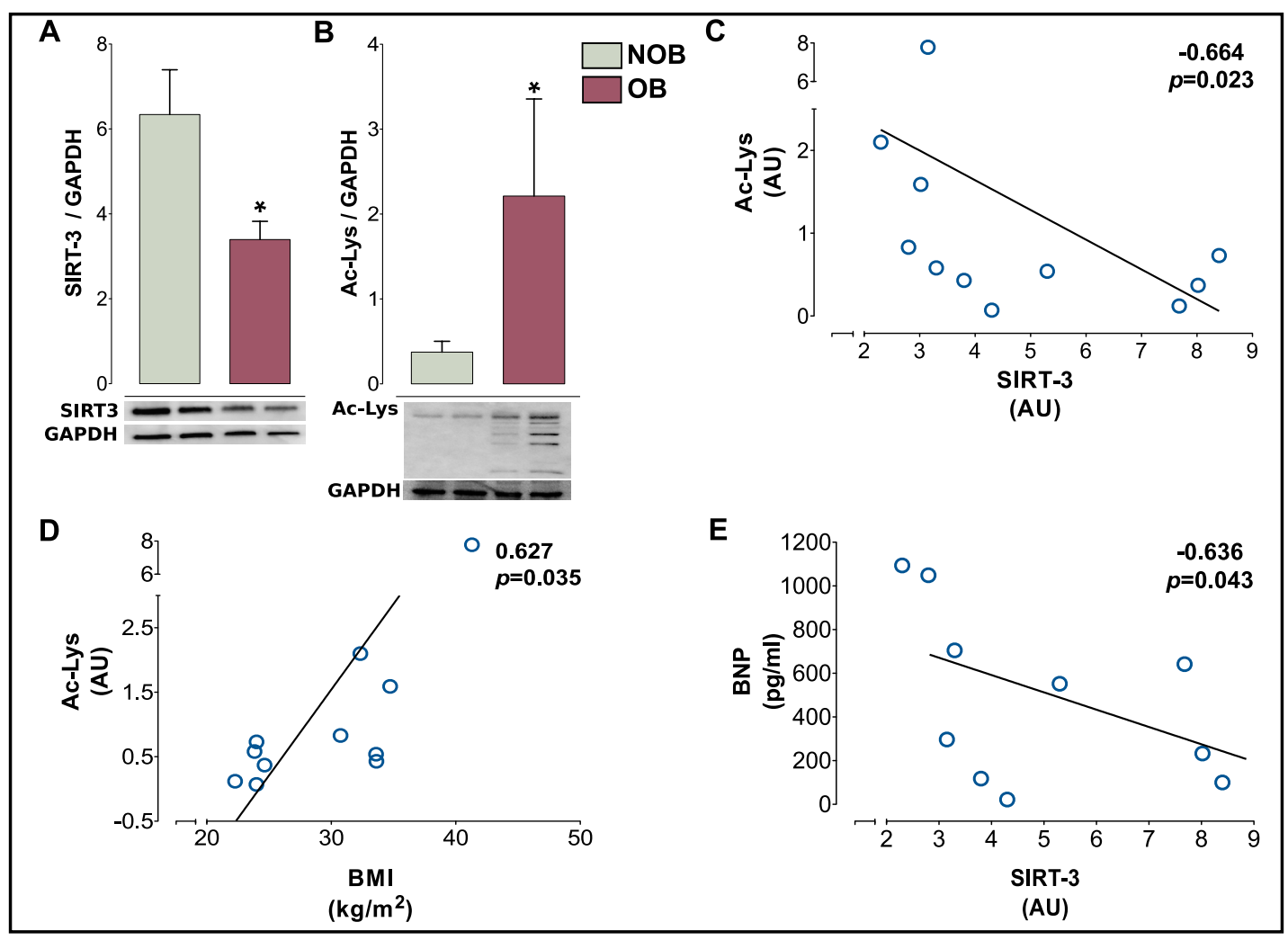

Fig. 1. Obesity is associated with SIRT3 down-regulation in HF patients. Tissue samples from non-obese (NOB) and obese (OB) patients with HF were isolated and protein expression of SIRT3 (A) and acetylation profile (B) was analyzed. GAPDH was used as a reference of protein expression. Correlation between (C) SIRT3 and acetylation profile, (D) BMI and acetylation profile, and (E) SIRT and BNP was performed. The data are presented as mean \pm SEM from NOB $(n=6)$ and OB $(n=5)$ patients. A t-test was performed to obtain the statistical analysis.

in comparison to its agematched CTL group. In the 12-months obese group, A-wave values decreased by $23 \%(p<0.001)$, E-values $28 \%(\mathrm{p}<0.0001)$, and the $\mathrm{E} / \mathrm{A}$ ratio $12 \%(\mathrm{p}<0.05)$. In addition, the deceleration time of these rats increased $45 \%$ ( $\mathrm{p}<0.0001)$ with respect to the control group, with a decrease in cardiac frequency. No changes in systolic parameter function were observed in either group. Nevertheless, the cardiac function of both obese groups was impaired; with a reduction in their mechanical performance index of 10 and 20\%, and 10 and $14 \%$ of the $\mathrm{MVO}_{2}$, respectively (Table 3). Furthermore, the rats in the 12-month obese group showed a reduction of phosphocreatine levels in the presence of isoproterenol (Fig. $2 \mathrm{~A}$ ) indicating that the energy homeostasis is being lost, reinforcing the idea of impaired cardiac function. Since the observed alterations in the $\mathrm{MVO}_{2}$ and ATP/PCr content may have been due to deficient mitochondrial oxidative metabolisms, we tested this possibility on isolated mitochondria from the 12-months obese rats. 
Table 3. Parameters of cardiac function in obese rat model. IVRT/RR: Isovolumetric relaxation time/R wave to R wave interval, MPI: Mechanical performance index, $+\mathrm{dP} / \mathrm{dt}$ and $-\mathrm{dP} / \mathrm{dt}$, maximal slope of the systolic pressure increment and diastolic pressure decrement, respectively; MVO2: myocardial oxygen consumption. Mean \pm SEM ${ }^{*} \mathrm{p}<0.05 ;{ }^{* *} \mathrm{p}<0.001 ;{ }^{* * *} \mathrm{p}<0.0001$ compared with the age-matched control group; $\mathrm{n}=17-22$. Metabolic and mechanical parameters $n=4-6$

\begin{tabular}{|c|c|c|c|c|}
\hline \multirow{2}{*}{ Parameters } & \multicolumn{2}{|c|}{6 months } & \multicolumn{2}{|c|}{12 months } \\
\hline & CTL & Obesity & CTL & Obesity \\
\hline \multicolumn{5}{|l|}{ Systolic function: } \\
\hline Fractional shortening (\%) & $61 \pm 1.3$ & $61.6 \pm 0.4$ & $62.9 \pm 0.6$ & $61.9 \pm 1$ \\
\hline Ejection fraction (\%) & $89 \pm 0.8$ & $89 \pm 0.4$ & $93 \pm 0.9$ & $93 \pm 1$ \\
\hline \multicolumn{5}{|l|}{ Diastolic function: } \\
\hline IVRT/RR (\%) & $0.19 \pm 0.009$ & $0.17 \pm 0.003$ & $0.18 \pm 0.004$ & $0.18 \pm 0.005$ \\
\hline E wave $(\mathrm{cm} / \mathrm{s})$ & $76 \pm 7$ & $79 \pm 4$ & $97 \pm 4$ & $70 \pm 4^{* * *}$ \\
\hline A wave $(\mathrm{cm} / \mathrm{s})$ & $71 \pm 6$ & $93 \pm 4^{*}$ & $85 \pm 4$ & $65 \pm 5^{* *}$ \\
\hline E/A ratio & $1.07 \pm 0.005$ & $0.85 \pm 0.03^{* * *}$ & $1.14 \pm 0.03$ & $1.07 \pm 0.06^{*}$ \\
\hline Deceleration time $(\mathrm{m})$ & $0.47 \pm 0.02$ & $0.57 \pm 0.02 *$ & $0.37 \pm 0.02$ & $0.53 \pm 0.03^{* * *}$ \\
\hline \multicolumn{5}{|l|}{ Hemodynamic parameters: } \\
\hline Heart rate (beats/min) & $415.4 \pm 12.8$ & $417.6 \pm 5.9$ & $424.6 \pm 10$ & $393.4 \pm 9.7^{*}$ \\
\hline End-systolic volume ( $\mu \mathrm{L} /$ beat) & $0.19 \pm 0.001$ & $0.27 \pm 0.01$ & $0.32 \pm 0.04$ & $0.27 \pm 0.02$ \\
\hline Cardiac output $(\mu \mathrm{L} / \mathrm{min})$ & $78.3 \pm 4.4$ & $83.6 \pm 4.4$ & $136.4 \pm 17.5$ & $104.1 \pm 6.8$ \\
\hline \multicolumn{5}{|l|}{ Mechanical parameters } \\
\hline MPI (mmHg.beat $\cdot$ min-1) (X1000) & $45.4 \pm 1.9$ & $40.8 \pm 1.2^{*}$ & $44.2 \pm 1.7$ & $35.4 \pm 4.2^{* *}$ \\
\hline$+\mathrm{dP} / \mathrm{dt}(\mathrm{x} 1000)$ & $6.5 \pm 2.4$ & $5.4 \pm 1.4$ & $5.8 \pm 0.9$ & $5.2 \pm 0.7$ \\
\hline$-\mathrm{dP} / \mathrm{dt}(\mathrm{x} 1000)$ & $3.2 \pm 0.7$ & $2.4 \pm 0.6$ & $3.4 \pm 0.5$ & $2.1 \pm 0.8^{* *}$ \\
\hline \multicolumn{5}{|l|}{ Metabolic parameters: } \\
\hline MVO2 ( $\mu$ mol02.g-1.min-1) & $29.7 \pm 0.8$ & $26.6 \pm 1.3^{*}$ & $27.2 \pm 1.7$ & $23.5 \pm 0.9^{* *}$ \\
\hline
\end{tabular}

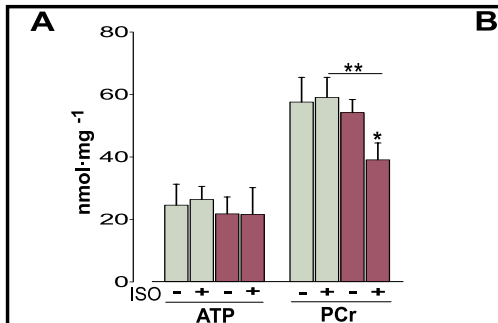

$\mathbf{C}$

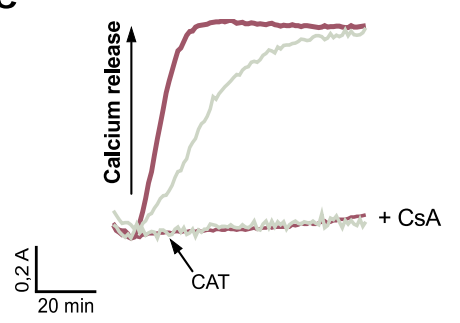

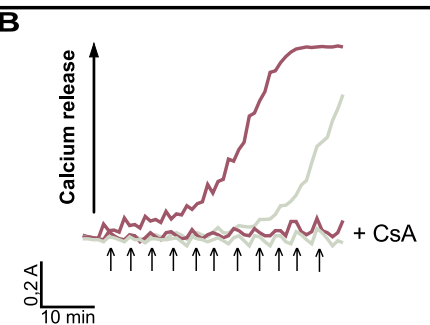

D

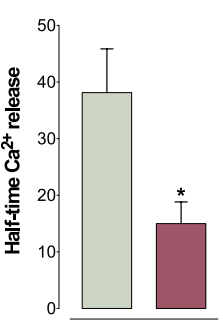

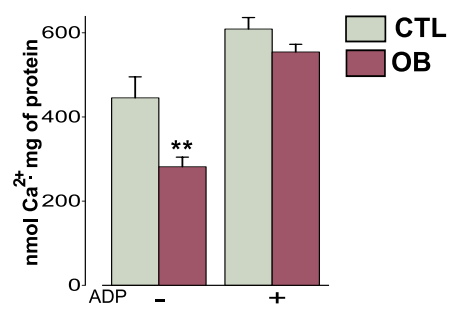

$\mathbf{E}$

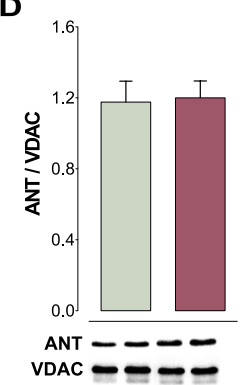

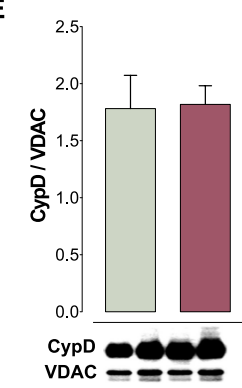

Fig. 2. Obesity promotes lost in the energy homeostasis and prone to permeability transition pore opening. (A) Mechanical performance index and energetic metabolites were evaluated in presence and absence of isoproterenol (ISO; data from 3 independent experiments) (B) Ca2+ retention capacity (CRC) of mitochondria in presence or absence of ADP and its representative trace of simultaneous measurements of crescent concentrations of $\mathrm{Ca} 2+$ with or without CsA. The arrow indicates the addition of $10 \mathrm{nM} \mathrm{Ca} 2+$. (C) Half-time Ca2+ release in the presence of CAT and ADP and a representative trace (data from 6 independent animals). Isolated mitochondria from hearts were analyzed for (D) ANT and (E) CypD expression by western blot; VDAC expression was used as a reference (data from 5 independent experiments). The error bars indicate the SEM. A t-test was performed to obtain the statistical analysis. Green bars indicate CTL groups and red bars 12 -mo OB groups. In A: *p<0.05 vs no ISO; In A-E: **p<0.01 vs CTL. 
SIRT3 deficiency and mitochondrial protein hyperacetylation stimulate permeability transition pore openings in rats with obesity

To assess the effects of these metabolic changes and cardiac functions at the mitochondrial level, we evaluated the mitochondrial respiratory rates. The respiratory control was not significantly affected. Remarkably, the hearts of the obese rats showed a $25 \%$ decrease in mitochondrial yield, suggesting an increase in mitochondrial fragility (Supplementary Table S2). We therefore assessed the MPTP opening sensitivity to the mitochondrial $\mathrm{Ca}^{2+}$ retention capacity (CRC). The CRC was significantly lower $(p<0.01)$ in the mitochondria of the obese group in comparison to CTL. The addition of ADP reestablished the $\mathrm{CRC}$, suggesting that the increased sensitivity of the mPTP to $\mathrm{Ca}^{2+}$ is favored by the activation of ANT in obese rats (Fig. 2B). Furthermore, we analyzed the half-time for the release of $\mathrm{Ca}^{2+}$ in the presence of carboxyatractyloside (CAT), which abolishes the ADP protection-inducing c-conformation of the ANT. The half-time to MPTP opening was found to be lower in the obese rats $(\mathrm{p}<0.05)$, whereas CsA completely inhibited the opening of the mPTP in both cases (Fig. 2C). These data confirm that metabolic changes in animals with obesity increase their sensitivity to increased opening of mPTP in heart tissues. To determine whether these changes respond to additional modifications other than SIRT3 deficiency and hyperacetylation of CypD, we explored the possible changes that occur in the ANT and CypD and found that the expression of both proteins remained unaltered in the heart mitochondria of the obese group in comparison to control (Fig. 2D and 2E). Based on this evidence, we decided to evaluate whether the changes observed in the sucrose-fed rats also increase oxidative stress for mitochondrial dysfunction and ANT oxidation (Fig. 3). We measured the aconitase activity as a marker of $\mathrm{O}_{2}^{-}$damage (Fig. 3A), the level of protein carbonylation (Fig. 3B), and the oxidation of the thiol groups (Fig. 3C) of the mitochondrial proteins, and we observed no significant changes in any of these parameters. Additionally, when we evaluated whether the interaction between ADP and ANT decreases with the oxidation of ANT, we found that the oxidation level of the ANT remained unchanged (Fig. $3 \mathrm{D}$ ), suggesting that susceptibility to the MPTP opening is not associated with the oxidation of the ANT's thiol residue. 
In order to ascertain the role of SIRT3 in the MPTP opening induced by obesity, we determined whether SIRT3, which is necessary to maintain the deacetylated state of CypD, is related to the affinity of CypD to ANT and the subsequent MPTP opening. The expression of SIRT3 and the acetylation status of mitochondrial proteins were evaluated in the obese and control rats (Fig. 4). We also evaluated the gene expression of the counterpart, the acetyltransferase GCN5L1 (Fig. 4A), in the obese rats and observed that it was up-regulated with respect to the control group (26\%; $\mathrm{p}<$ 0.05). Accordingly, SIRT3 protein expression was down-regulated in the obese group (35\%; p < 0.05 ; Fig. 4B). These data were in accordance with the observed hyperacetylated mitochondrial protein profile ( $\mathrm{p}=0.006$; Fig. $4 \mathrm{C})$ and specifically the CypD acetylation shown by the im munoprecipitation experiments $(\mathrm{p}=0.035$; Fig. 4D).

\section{Discussion}

In this study, we showed that obesity and MetSinduce SIRT3 deficiency, and as a consequence, CypD is hyperacetylated, favoring the MPTP opening and ventricular cardiac dysfunction. The metabolic alterations seen in our experimental model are in accordance with the clinical criteria of impairments in ventricular and mitochondrial functioning [18, 19]. Of note even when human subjects have reduced $\mathrm{EF}$ and the rats demonstrate preserved EF, it has been shown that the HF related to obesity tends to be associated with preserved EF in accordance with our model $[20,21]$. Importantly, the findings from the obese and MetS animal models correlated with the changes in the acetylation profile of the end-stage failing hearts of humans, suggesting that mitochondrial dysfunction is partially mediated by SIRT3dependent hyperacetylation.

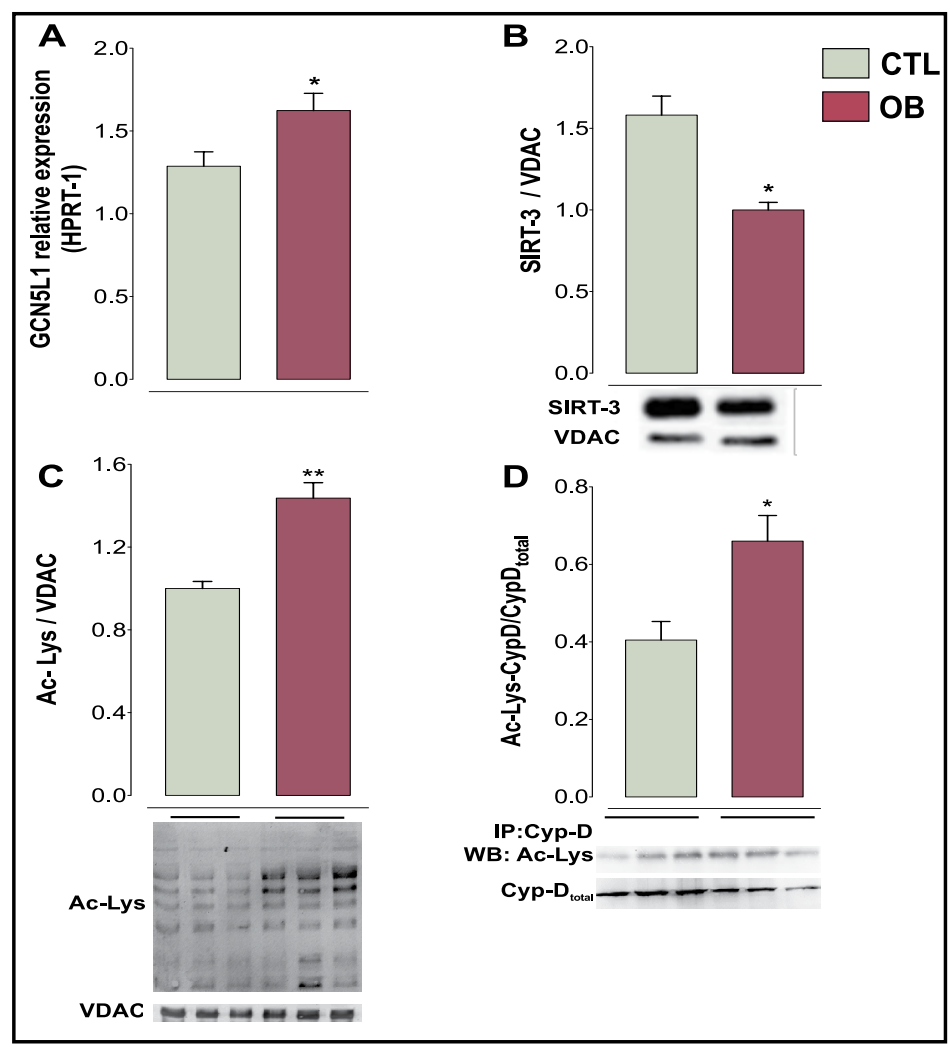

Fig. 4. SIRT3 down-regulation is associated with CypD acetylation in OB model. Hearts from rats with 12 months of obesity and MetS (OB) were isolated for a total gene expression of (A) GCN5L1. The gene expression levels were normalized to HPRT-1 gene expression and are presented as the relative expression. (B) Mitochondria were isolated from hearts of CTL or OB rats and analyzed for SIRT3 expression by western blot; VDAC expression was used as a reference. (C) Acetylation profile from cardiac mitochondria was analyzed by western blotting; VDAC was used as a reference. (D) Immunoprecipitation (IP) of protein extracts from heart mitochondria with monoclonal anti-CypD followed by immunoblot analysis with the anti-Ac-Lys antibody. Total CypD was used as control. The data are presented as mean \pm SEM from at least 6 independent experiments for gene expression and 5 for protein levels. A t-test was performed to obtain the statistical analysis. Green bars indicate CTL groups and red bars OB groups. ${ }^{*} \mathrm{p}<0.035$ and ${ }^{* *} \mathrm{p}<0.006$ vs CTL. 


\section{Cellular Physiology Cell Physiol Biochem 2019;53:465-479 \\ \begin{tabular}{ll|l} 
and Biochemistry & $\begin{array}{l}\text { DOl: 10.33594/000000151 } \\
\text { Published online: } 30 \text { August } 2019\end{array}$ & $\begin{array}{l}\text { O } 2019 \text { The Author(s). Published by } \\
\text { Cell Physiol Biochem Press GmbH\&Co. KG }\end{array}$ \\
\cline { 2 - 3 } & Caslo
\end{tabular} \\ Castillo et al.: SIRT3 \& Acetylation in Failing Heart with Obesity}

Obesity and MetS induce energetic impairment and mitochondrial dysfunction

In the present study, we observed that our obese animals developed significant weight gain, visceral fat accumulation, and insulin resistance similar to the results of previous reports [2]. Moreover, it has been reported that these rats develop ventricular dysfunction and susceptibility to deadly arrhythmias [15]. Interestedly, a similar model in mice also found that a diet rich in sugar induces arrhythmias and cardiac dysfunction correlating with mitochondrial dysfunction [22] that is possibly dependent on mitochondrial and cytosolic calcium handling [15]. In this regard, recent results among cardiomyocytes and animals support the hypothesis that mitochondrial $\mathrm{Ca}^{2+}$ overload is a negative consequence of diabetic cardiomyopathy, and increasing the expression of mitochondrial calcium channels could improve cardiac function [23]. On the other hand, sucrose-fed models have also shown impairments in cardiac cytosolic $\mathrm{Ca}^{2+}$ handling, possibly related to sarcoplasmic reticulum malfunction [24]. Consequently, an increased cytosolic $\mathrm{Ca}^{2+}$ concentration disrupts mitochondrial membrane potential $(\Delta \Psi \mathrm{m})$ and induces the opening of mPTP $[4,5]$. Here, we propose that mitochondrial dysfunction in obese and MetS is dependent on an increased susceptibility of the mPTP opening, and we observed an increase in permeability transition in the 12-month obese group that was associated with CypD and assessed using a CRC analysis (Fig. 1B). CypD is a mitochondrial matrix protein with peptidyl-prolyl isomerase activity that functions as a main regulator of an mPTP $[6,25]$. For instance, CypD ${ }^{\%}$ in mice exhibits protection against mitochondrial damage provoked by ischemia/reperfusion and $\mathrm{Ca}^{2+}$ overloading, whereas cardiomyocytes of rats with CypD overexpression are hypersensitive to these stimuli and show early mitochondrial dysfunction and cell death $[6,25]$. The protection against cell death in $\mathrm{CypD}^{-/}$models is seen only when the stressors are $\mathrm{Ca}^{2+}$ overload and oxidative stress; whereas in cell death mediated by the Bcl-2 family members, $\mathrm{CypD}^{-/}$appears to have only a secondary role, if any [25]. One proposed mechanism through which CypD induces an mPTP opening indicates the binding of CypD to the ANT [7], and can be inhibited by CsA [26] (as observed in Fig. 1C). In this regard, we evaluated whether the increased susceptibility to the mPTP opening was due to oxidative stress, and we observed that just as the expression levels of ANT and CypD remain intact, cardiac protein oxidation did not increase in response to obesity or the presence of an MetS. These results anthologize with other reports of obese and MetS animals, in which cardiac mitochondria showed increased $\mathrm{H}_{2} \mathrm{O}_{2}$ production and superoxide radicals [27]. This is possibly due to the differences between the species (rat and mice) or the time of treatment (2-4 months), because it has been observed in the hearts of aged rats that the activities of the main oxygen-radical scavenger enzymes are stimulated in the mitochondria as a compensatory mechanism [28].

\section{SIRT3-dependent hyperacetylation in failing human hearts}

The binding of CypD to the inner membrane components is positively correlated to its acetylation state in the mitochondrial matrix $[9,29]$. Indeed, it has been shown that CypD deacetylation is critical for regulating mPTP opening, avoiding mitochondrial transition and cell death in ischemic postconditioning, and preventing reperfusion injury [29]. The acetylation profile of mitochondrial proteins increases with the expression and activity of GCN5L1 [30], whereas SIRT3 counteracts this effect through its NAD dependent deacetylase activity [9]. Accordingly, SIRT3 down-regulation in mice with chronic high fat diets correlates with more severe MetS and hyperacetylated proteins [10] that promotes fatty acid oxidation $[11,31]$. In addition, CypD acetylation was associated with SIRT3 downregulation after HF induction in diabetic rats [13]. These data suggest that chronic metabolic changes must occur in order to observe SIRT3 downregulation. While we did not assess SIRT4 and SIRT5 expression, the other mitochondrial acetylases, previous works have shown that these sirtuins do not participate in lysine acetylation [32,33]. Furthermore, it has been shown in cardiomyocytes from SIRT3 knockout mice; that mitochondrial swelling due to increases in the mPTP opening with hypersensitivity to cardiac stress [9] is in line with our findings and occurs despite SIRT4 compensatory upregulation [33]. In our model, we demonstrate a decrease in SIRT3 expression, after chronic metabolic changes induced by sucrose ingestion 


\section{Cellular Physiology Cell Physiol Biochem 2019;53:465-479 \\ \begin{tabular}{ll|l} 
and Biochemistry $\begin{array}{l}\text { DOl: 10.33594/000000151 } \\
\text { Published online: } 30 \text { August } 2019\end{array}$ & $\begin{array}{l}\text { C } 2019 \text { The Author(s). Published by } \\
\text { Cell Physiol Biochem Press GmbH\&Co. KG }\end{array}$ \\
\hline
\end{tabular} \\ Castillo et al.: SIRT3 \& Acetylation in Failing Heart with Obesity}

for 12 months that was associated with the up-regulation of its opponent GCN5L1 compared to non-obese (Control-CTL) group. As expected, the CypD acetylation profile increased, correlating with the augmented sensitivity to the MPTP opening and heart dysfunction.

In line with these observations and because obesity, and in particular BMI, is associated with MetS, diabetes and HF [34], we sought to determine whether patients with HF and obesity display hyperacetylation profiles and a deregulation in SIRT3 protein expression in contrast to non-obese HF patients, both at the advanced stages of the disease and aged 55 years old on average. We observed for the first time that even at this stage of the disease, SIRT3 expression remains lower in obese than non-obese patients in which BMI positively correlates with acetylation profile. This could be explained by the evidence that chronic metabolic changes are involved in SIRT3 downregulation though the suppression of the transcription coactivator PGC-1 $\alpha$, that has been proposed as a key regulator of energy metabolism [10]. Moreover, SIRT3 downregulation has also been associated with DNA sequence variants and SNPs that decrease SIRT3 promoter activity, in patients with MetS and myocardial infarction [35]. Although we were not able to analyze the specific mitochondrial hyperacetylation or its dependence on PGC- $1 \alpha$ activation, the significant changes observed in SIRT3 expression and acetylation were able to be observed in a scarce number of evaluated patients in which BNP levels were elevated and negatively correlated with SIRT3 expression. This suggests that chronic metabolic changes that are in association to BMI [36] may contribute to more cardiac damage associated to SIRT3 downregulation.

SIRT3 deacetylase is involved in the deacetylation of approximately 151 peptides of 48 mitochondrial proteins. In addition, CypD, a substrate of SIRT3, has been also associated with the regulation of the acetylation of enzymes that differ from those targeted by SIRT3 [37]. This SIRT3- and CypD-dependent acetylation has been associated with cardioprotection $[6,29]$. Regarding the importance of acetylation in the regulation of the metabolism and $\mathrm{HF}$, both CypD ${ }^{-/-}$and SIRT3 ${ }^{-/-}$mice display an increased acetylation of proteins in fatty acid oxidation [37] altering the mitochondrial bioenergetics by promoting glucose metabolism, indicating that CypD plays physiological roles other than mPTP opening [38]. Myocardial acetyl-proteomics identified thousands of mitochondrial acetylation sites in end-stage failing human hearts [39] as well as in SIRT3\% mice [10]. Most mitochondrial hyperacetylated sites have been previously identified as SIRT3 targets $[9,10]$, and particularly, the hyperacetylation of CypD and other mPTP regulators have been associated with cardiac dysfunction $[9,40]$. Moreover, mitochondrial lysine acetylation proteins by $\mathrm{NAD}^{+} / \mathrm{NADH}$ ratio impairment have been also associated [40], indicating that targeting the imbalance in energetic metabolism is crucial to avoid cardiomyocytes' detrimental functions that lead to HF.

\section{Modulation of hyperacetylation as a potential therapy for heart failure}

As SIRT3 activity depends on NAD ${ }^{+}$levels, which are often reduced in MetS and HF [40, 41], $\mathrm{NAD}^{+}$repletion therapies avoid SIRT3 down-regulation, show a normalized acetylation state of proteins such as CypD, and demonstrate improved outcomes in HF mouse models $[10,40,42]$, even after short-term administration [43]. Therefore, the modulation of protein acetylation is considered a novel therapeutic strategy for combating MetS and cardiovascular diseases $[40,44]$. In this context, preliminary data suggests that $\mathrm{NAD}^{+}$levels can be normalized in humans with no adverse effects from nicotinamide riboside supplementation [45].

Until now, the accepted mechanisms by which sirtuin activity can be significantly stimulated are calorie restrictions and strenuous exercise, that are difficult to achieve in a clinical setting. Thus the research of natural molecules such as cardioprotective agents has increased, and several phytochemicals (phenolic compounds), such as resveratrol, honokiol, and oroxylin A, have been described as positive SIRT3 regulators [44]. Even though these polyphenol compounds have shown promising results as SIRT3 activators in experimental models, pharmacokinetic studies have indicated that their bioavailability is extremely low, which may blunt their protective effect in a clinical setting [46]. Consequently, the successful use of natural compounds for cardiac therapy requires the development of effective drug delivery systems [47]. Recently, endothelial dysfunction in HF has been explored as a 


\section{Cellular Physiology Cell Physiol Biochem 2019;53:465-479 \\ \begin{tabular}{c|c|c|c|}
\hline DOI: 10.33594/000000151 2019 The Author(s). Published by \\
and Biochemistry
\end{tabular} \\ \begin{tabular}{ll} 
Published online: 30 August 2019 Cell Physiol Biochem Press GmbH\&Co. KG \\
\hline
\end{tabular} \\ Castillo et al.: SIRT3 \& Acetylation in Failing Heart with Obesity}

novel avenue for the delivery of nanocarrier-based therapies to reach the heart through a dysfunctional permeable endothelium [48]. In a HF murine model, the passive cardiac accumulation of nanocarriers was 15-fold higher in the HF mice compared to control [49]. This approach should be used as a potential avenue for SIRT3 activator therapy, potentially leading to other novel therapies that may improve heart dysfunction in patients.

\section{Conclusion}

Here, we suggest that conditions such as obesity and MetS induce SIRT3 down-regulation, which leads to changes in the acetylation profile of mitochondrial proteins such as CypD that result in increases in mPTP opening and cardiac dysfunction. Remarkably, we showed that obese HF patients exhibit more lysine hyperacetylated proteins associated with BMI and less SIRT3 expression. Together, these findings support the importance of mitochondria in HF development. They highlight the necessity of a mitochondrial therapeutic approach to reestablish SIRT3 expression/activity, not only as a potential therapy for HF patients as has been suggested, but also to prevent cardiovascular disease in patients with obesity and MetS.

\section{Acknowledgements}

We thank Edgar Acuña, MD for his exceptional technical assistance, Flor Morales, MSc and Andrea Cordero, MD for her help with sample selection. We thank Drs. Leticia Elizondo and Salvador Uribe for their helpful discussion.

This work was partially supported by Cardiovascular Medicine Research GroupTecnológico de Monterrey 0020CAT131 as well as CONACYT-México grants 151136 and 256577 (G. García-Rivas). JAM, NT-S, JB-R and AT-Q were supported by CONACYT graduate scholarships. ECC, HC-V and E G-B were supported by postdoctoral Fellowship (at GarcíaRivas lab) from CONACYT.

Authors' contributions: Conception and design of the research: ECC, HC-V, JAM, GG-R; Acquisition of the data: ECC, HC-V, JAM, C-SP, EG-B, JB-R, AT-Q, NG, N-TS, KY; Analysis and interpretation of the data: ECC, H-CV, JAM, C-SP, EG-B, JB-R, AT-Q, NG, N-TS, KY; Statistical analysis: ECC, HC-V, JAM, C-SP, EG-B, JB-R, AT-Q, NG; Obtaining founding and supervising the work: GG-R; Drafting the manuscript: ECC, HC-V, JAM, GG-R, GT-A, N-TS; Critical revision of the manuscript for important intellectual content: ECC, HC-V, C-SP, EG-B, GG-R, GT-A, KY.

\section{Disclosure Statement}

The authors have no conflicts of interest to declare.

\section{References}

1 Wilson PWF, D’Agostino RB, Parise H, Sullivan L, Meigs JB: Metabolic syndrome as a precursor of cardiovascular disease and type 2 diabetes mellitus. Circulation 2005;112:3066-3072.

2 Carvajal K, El Hafidi M, Marin-Hernández A, Moreno-Sánchez R: Structural and functional changes in heart mitochondria from sucrose-fed hypertriglyceridemic rats. Biochim Biophys Acta 2005;1709:231-239.

3 Ge F, Hu C, Hyodo E, Arai K, Zhou S, Lobdell H, Walewski JL, Homma S, Berk PD: Cardiomyocyte triglyceride accumulation and reduced ventricular function in mice with obesity reflect increased long chain Fatty Acid uptake and de novo Fatty Acid synthesis. J Obes 2012;2012:205648.

4 García-Rivas G de J, Carvajal K, Correa F, Zazueta C: Ru360, a specific mitochondrial calcium uptake inhibitor, improves cardiac post-ischaemic functional recovery in rats in vivo. Br J Pharmacol 2006;149:829-837. 


\section{Cellular Physiology Cell Physiol Biochem 2019;53:465-479 \begin{tabular}{ll|l|l|l}
\hline DOI: 10.33594/000000151 & 2019 The Author(s). Published by
\end{tabular} and Biochemistry Published online: 30 August 2019 Cell Physiol Biochem Press GmbH\&Co. KG \\ Castillo et al.: SIRT3 \& Acetylation in Failing Heart with Obesity}

5 Oropeza-Almazán Y, Vázquez-Garza E, Chapoy-Villanueva H, Torre-Amione G, García-Rivas G: Small Interfering RNA Targeting Mitochondrial Calcium Uniporter Improves Cardiomyocyte Cell Viability in Hypoxia/Reoxygenation Injury by Reducing Calcium Overload. Oxid Med Cell Longev 2017;2017:5750897.

6 Baines CP, Kaiser RA, Purcell NH, Blair NS, Osinska H, Hambleton MA, Brunskill EW, Sayen MR, Gottlieb RA, Dorn GW, Robbins J, Molkentin JD: Loss of cyclophilin D reveals a critical role for mitochondrial permeability transition in cell death. Nature 2005;434:658-662.

7 Halestrap AP, Brenner C: The adenine nucleotide translocase: a central component of the mitochondrial permeability transition pore and key player in cell death. Curr Med Chem 2003;10:1507-1525.

8 Giorgio V, von Stockum S, Antoniel M, Fabbro A, Fogolari F, Forte M, Glick GD, Petronilli V, Zoratti M, Szabó I, Lippe G, Bernardi P: Dimers of mitochondrial ATP synthase form the permeability transition pore. Proc Natl Acad Sci USA 2013;110:5887-5892.

9 Hafner AV, Dai J, Gomes AP, Xiao CY, Palmeira CM, Rosenzweig A, Sinclair DA: Regulation of the mPTP by SIRT3-mediated deacetylation of CypD at lysine 166 suppresses age-related cardiac hypertrophy. Aging (Albany NY) 2010;2:914-923.

10 Hirschey MD, Shimazu T, Jing E, Grueter CA, Collins AM, Aouizerat B, Stančáková A, Goetzman E, Lam MM, Schwer B, Stevens RD, Muehlbauer MJ, Kakar S, Bass NM, Kuusisto J, Laakso M, Alt FW, Newgard CB, Farese RV Jr, Kahn CR, et al.: SIRT3 deficiency and mitochondrial protein hyperacetylation accelerate the development of the metabolic syndrome. Mol Cell 2011;44:177-190.

11 Thapa D, Zhang M, Manning JR, Guimarães DA, Stoner MW, O’Doherty RM, Shiva S, Scott I: Acetylation of mitochondrial proteins by GCN5L1 promotes enhanced fatty acid oxidation in the heart. Am J Physiol Heart Circ Physiol 2017;313:H265-H274.

12 Yang L, Zhang J, Xing W, Zhang X, Xu J, Zhang H, Chen L, Ning X, Ji G, Li J, Zhao Q Gao F: SIRT3 Deficiency Induces Endothelial Insulin Resistance and Blunts Endothelial-Dependent Vasorelaxation in Mice and Human with Obesity. Sci Rep 2016;6:23366.

13 Parodi-Rullan R, Barreto-Torres G, Ruiz L, Casasnovas J, Javadov S: Direct renin inhibition exerts an antihypertrophic effect associated with improved mitochondrial function in post-infarction heart failure in diabetic rats. Cell Physiol Biochem 2012;29:841-850.

14 Willis BC, Salazar-Cantú A, Silva-Platas C, Fernández-Sada E, Villegas CA, Rios-Argaiz E, González-Serrano P, Sánchez LA, Guerrero-Beltrán CE, García N, Torre-Amione G, García-Rivas GJ, Altamirano J: Impaired oxidative metabolism and calcium mishandling underlie cardiac dysfunction in a rat model of post-acute isoproterenol-induced cardiomyopathy. Am J Physiol Heart Circ Physiol 2015;308:H467-H477.

15 Fernández-Sada E, Silva-Platas C, Villegas CA, Rivero SL, Willis BC, García N, Garza JR, Oropeza-Almazán Y, Valverde CA, Mazzocchi G, Zazueta C, Torre-Amione G, García-Rivas G: Cardiac responses to $\beta$-adrenoceptor stimulation is partly dependent on mitochondrial calcium uniporter activity. Br J Pharmacol 2014;171:4207-4221.

16 Silva-Platas C, García N, Fernández-Sada E, Dávila D, Hernández-Brenes C, Rodríguez D, García-Rivas G: Cardiotoxicity of acetogenins from Persea americana occurs through the mitochondrial permeability transition pore and caspase-dependent apoptosis pathways. J Bioenerg Biomembr 2012;44:461-471.

17 Fernández-Sada E, Torres-Quintanilla A, Silva-Platas C, García N, Willis BC, Rodríguez-Rodríguez C, De la Peña E, Bernal-Ramírez J, Treviño-Saldaña N, Oropeza-Almazán Y, Castillo EC, Elizondo-Montemayor L, Carvajal K, García-Rivas G: Proinflammatory Cytokines Are Soluble Mediators Linked with Ventricular Arrhythmias and Contractile Dysfunction in a Rat Model of Metabolic Syndrome. Oxid Med Cell Longev 2017;2017:7682569.

18 Aijaz B, Ammar KA, Lopez-Jimenez F, Redfield MM, Jacobsen SJ, Rodeheffer RJ: Abnormal cardiac structure and function in the metabolic syndrome: a population-based study. Mayo Clin Proc 2008;83:1350-1357.

19 Gray S, Kim JK: New insights into insulin resistance in the diabetic heart. Trends Endocrinol Metab 2011;22:394-403.

20 Oktay AA, Rich JD, Shah SJ: The Emerging Epidemic of Heart Failure with Preserved Ejection Fraction. Curr Heart Fail Rep 2013;10:401-410.

21 Packer M, Kitzman DW: Obesity-Related Heart Failure with a Preserved Ejection Fraction: The Mechanistic Rationale for Combining Inhibitors of Aldosterone, Neprilysin, and Sodium-Glucose Cotransporter-2. JACC Heart Fail 2018;6:633-639. 


\section{Cellular Physiology Cell Physiol Biochem 2019;53:465-479 \begin{tabular}{ll|l|l|}
\hline DOI: 10.33594/000000151 & (O 2019 The Author(s). Published by \\
\hline
\end{tabular} and Biochemistry Published online: 30 August 2019 Cell Physiol Biochem Press GmbH\&Co. KG \\ Castillo et al.: SIRT3 \& Acetylation in Failing Heart with Obesity}

22 Federico M, Portiansky EL, Sommese L, Alvarado FJ, Blanco PG, Zanuzzi CN, Dedman J, Kaetzel M, Wehrens XHT, Mattiazzi A, Palomeque J: Calcium-calmodulin-dependent protein kinase mediates the intracellular signalling pathways of cardiac apoptosis in mice with impaired glucose tolerance. J Physiol 2017;595:4089-4108.

23 Suarez J, Cividini F, Scott BT, Lehmann K, Diaz-Juarez J, Diemer T, Dai A, Suarez JA, Jain M, Dillmann WH: Restoring mitochondrial calcium uniporter expression in diabetic mouse heart improves mitochondrial calcium handling and cardiac function. J Biol Chem 2018;293:8182-8195.

24 Vasanji Z, Cantor EJF, Juric D, Moyen M, Netticadan T: Alterations in cardiac contractile performance and sarcoplasmic reticulum function in sucrose-fed rats is associated with insulin resistance. Am J Physiol Cell Physiol 2006;291:C772-780.

25 Nakagawa T, Shimizu S, Watanabe T, Yamaguchi O, Otsu K, Yamagata H, Inohara H, Kubo T, Tsujimoto Y: Cyclophilin D-dependent mitochondrial permeability transition regulates some necrotic but not apoptotic cell death. Nature 2005;434:652-658.

26 Argaud L, Gateau-Roesch 0, Raisky 0, Loufouat J, Robert D, Ovize M: Postconditioning inhibits mitochondrial permeability transition. Circulation 2005;111:194-197.

27 Sverdlov AL, Elezaby A, Qin F, Behring JB, Luptak I, Calamaras TD, Siwik DA, Miller EJ, Liesa M, Shirihai OS, Pimentel DR, Cohen RA, Bachschmid MM, Colucci WS: Mitochondrial Reactive Oxygen Species Mediate Cardiac Structural, Functional, and Mitochondrial Consequences of Diet-Induced Metabolic Heart Disease. J Am Heart Assoc 2016;5:pii:e002555.

28 Muscari C, Giaccari A, Giordano E, Clô C, Guarnieri C, Caldarera CM. Role of reactive oxygen species in cardiovascular aging. Mol Cell Biochem 1996; 160-161:159-166.

29 Bochaton T, Crola-Da-Silva C, Pillot B, Villedieu C, Ferreras L, Alam MR, Thibault H, Strina M, Gharib A, Ovize $\mathrm{M}$, Baetz D: Inhibition of myocardial reperfusion injury by ischemic postconditioning requires sirtuin 3-mediated deacetylation of cyclophilin D. J Mol Cell Cardiol 2015;84:61-69.

30 Scott I, Webster BR, Li JH, Sack MN: Identification of a molecular component of the mitochondrial acetyltransferase programme: a novel role for GCN5L1. Biochem J 2012;443:655-661.

31 Alrob OA, Sankaralingam S, Ma C, Wagg CS, Fillmore N, Jaswal JS, Sack MN, Lehner R, Gupta MP, Michelakis ED, Padwal RS, Johnstone DE, Sharma AM, Lopaschuk GD: Obesity-induced lysine acetylation increases cardiac fatty acid oxidation and impairs insulin signalling. Cardiovasc Res 2014;103:485-497.

32 Lombard DB, Alt FW, Cheng HL, Bunkenborg J, Streeper RS, Mostoslavsky R, Kim J, Yancopoulos G, Valenzuela D, Murphy A, Yang Y, Chen Y, Hirschey MD, Bronson RT, Haigis M, Guarente LP, Farese RV Jr, Weissman S, Verdin E, Schwer B: Mammalian Sir2 Homolog SIRT3 Regulates Global Mitochondrial Lysine Acetylation. Mol Cell Biol 2007;27:8807-8814.

33 Parodi-Rullán RM, Chapa-Dubocq X, Rullán PJ, Jang S, Javadov S: High Sensitivity of SIRT3 Deficient Hearts to Ischemia-Reperfusion Is Associated with Mitochondrial Abnormalities. Front Pharmacol 2017;8:275.

34 Arnlöv J, Sundström J, Ingelsson E, Lind L: Impact of BMI and the metabolic syndrome on the risk of diabetes in middle-aged men. Diabetes Care 2011;34:61-65.

35 Yin X, Pang S, Huang J, Cui Y, Yan B: Genetic and Functional Sequence Variants of the SIRT3 Gene Promoter in Myocardial Infarction. PLoS One 2016;11:e0153815.

36 Moore SC, Matthews CE, Sampson JN, Stolzenberg-Solomon RZ, Zheng W, Cai Q, Tan YT, Chow WH, Ji BT, Liu DK, Xiao Q, Boca SM, Leitzmann MF, Yang G, Xiang YB, Sinha R, Shu XO, Cross AJ: Human metabolic correlates of body mass index. Metabolomics 2014;10:259-269.

37 Nguyen TTM, Wong R, Menazza S, Sun J, Chen Y, Wang G, Gucek M, Steenbergen C, Sack MN, Murphy E: Cyclophilin D modulates mitochondrial acetylome. Circ Res 2013;113:1308-1319.

38 Tavecchio M, Lisanti S, Bennett MJ, Languino LR, Altieri DC: Deletion of Cyclophilin D Impairs $\beta$-Oxidation and Promotes Glucose Metabolism. Sci Rep 2015;5:15981.

39 Horton JL, Martin OJ, Lai L, Riley NM, Richards AL, Vega RB, Leone TC, Pagliarini DJ, Muoio DM, Bedi KC Jr, Margulies KB, Coon JJ, Kelly DP: Mitochondrial protein hyperacetylation in the failing heart. JCI Insight 2016;2:pii:e84897.

40 Lee CF, Chavez JD, Garcia-Menendez L, Choi Y, Roe ND, Chiao YA, Edgar JS, Goo YA, Goodlett DR, Bruce JE, Tian R: Normalization of NAD+ Redox Balance as a Therapy for Heart Failure. Circulation 2016;134:883894. 


\section{Cellular Physiology Cell Physiol Biochem 2019;53:465-479

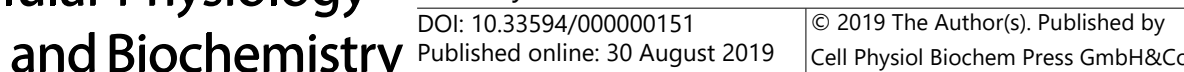 \\ Castillo et al.: SIRT3 \& Acetylation in Failing Heart with Obesity}

41 Escande C, Nin V, Price NL, Capellini V, Gomes AP, Barbosa MT, O'Neil L, White TA, Sinclair DA, Chini EN: Flavonoid Apigenin Is an Inhibitor of the NAD+ase CD38. Diabetes 2013;62:1084-1093.

42 Diguet N, Trammell SAJ, Tannous C, Deloux R, Piquereau J, Mougenot N, Gouge A, Gressette M, Manoury B, Blanc J, Breton M, Decaux JF, Lavery GG, Baczkó I, Zoll J, Garnier A, Li Z, Brenner C, Mericskay M: Nicotinamide Riboside Preserves Cardiac Function in a Mouse Model of Dilated Cardiomyopathy. Circulation 2018;137:2256-2273.

43 Zhang R, Shen Y, Zhou L, Sangwung P, Fujioka H, Zhang L, Liao X: Short-term administration of Nicotinamide Mononucleotide preserves cardiac mitochondrial homeostasis and prevents heart failure. J Mol Cell Cardiol 2017;112:64-73.

44 Treviño-Saldaña N, García-Rivas G: Regulation of Sirtuin-Mediated Protein Deacetylation by Cardioprotective Phytochemicals. Oxid Med Cell Longev 2017;2017:1750306.

45 Airhart SE, Shireman LM, Risler LJ, Anderson GD, Nagana Gowda GA, Raftery D, Tian R, Shen DD, O’Brien KD: An open-label, non-randomized study of the pharmacokinetics of the nutritional supplement nicotinamide riboside (NR) and its effects on blood NAD+ levels in healthy volunteers. PLoS One 2017;12:e0186459.

46 Walle T, Hsieh F, DeLegge MH, Oatis JE, Walle UK: High absorption but very low bioavailability of oral resveratrol in humans. Drug Metab Dispos 2004;32:1377-1382.

47 Lozano O, Torres-Quintanilla A, García-Rivas G: Nanomedicine for the cardiac myocyte: Where are we? J Control Release 2018;271:149-165.

48 Howitz KT, Bitterman KJ, Cohen HY, Lamming DW, Lavu S, Wood JG, Zipkin RE, Chung P, Kisielewski A, Zhang LL, Scherer B, Sinclair DA: Small molecule activators of sirtuins extend Saccharomyces cerevisiae lifespan. Nature 2003;425:191-196.

49 Ruiz-Esparza GU, Segura-Ibarra V, Cordero-Reyes AM, Youker KA, Serda RE, Cruz-Solbes AS, Amione-Guerra J, Yokoi K, Kirui DK, Cara FE, Paez-Mayorga J, Flores-Arredondo JH, Guerrero-Beltrán CE, Garcia-Rivas G, Ferrari M, Blanco E, Torre-Amione G: A specifically designed nanoconstruct associates, internalizes, traffics in cardiovascular cells, and accumulates in failing myocardium: a new strategy for heart failure diagnostics and therapeutics. Eur J Heart Fail 2016;18:169-178. 